\title{
Three approaches to quantitative health impact assessment in Copenhagen
}

Holm, Astrid Ledgaard; Brønnum-Hansen, Henrik; Diderichsen, Finn

Published in:

European Journal of Public Health

Publication date:

2014

Document license:

Unspecified

Citation for published version (APA):

Holm, A. L., Brønnum-Hansen, H., \& Diderichsen, F. (2014). Three approaches to quantitative health impact assessment in Copenhagen. European Journal of Public Health, Vol. 24(Supplement 2, 2014), $20-21$. 
Three approaches to quantitative health impact assessment in Copenhagen Astrid Ledgaard Holm

AL Holm, H Brønnum-Hansen, F Diderichsen Section of Social Medicine, Department of Public Health, University of Copenhagen, Copenhagen, Denmark Contact: asho@sund.ku.dk

We present examples of the use of (quantitative) health impact assessment (HIA) in Copenhagen, Denmark. Three methodologies for quantitative HIA were adapted and applied to analyse the health impacts of different intervention scenarios targeting a range of determinants of health in the population of Copenhagen:

1. Assessment of the overall health effects of replacing travel by car to and from work or place of education in Copenhagen with active transportation by bicycle. The potential health effects of changes in physical activity, exposure to air pollution and risk of traffic accidents were included in the analysis. The results showed that increased commuting by bicycle could reduce the burden of disease in the study population.

2. Estimation of the potential health impacts of decreased smoking prevalence in Copenhagen. An adapted version of the DYNAMO-HIA model (DYNAmic MOdelling for Health Impact Assessment) was used to estimate the potential health effects. Four intervention scenarios were compared to a reference scenario: Decreased smoking initiation among youth; Increased quit rates among current smoking adults; Decreased re-initiation rates among former smoking adults; A combination of these factors. The 
assessment showed that the largest health effects were obtained through the combined scenario, which targeted several population sub-groups with different risk profiles.

3. Estimation of the potential health effects and costs of changes in alcohol consumption. The analyses illustrate the link between HIA and health economic evaluation, and an adapted version of the methods used in the large Australian Assessing Cost-Effectiveness in Prevention (ACE-prevention) study was applied. The results showed that most structural interventions to decrease alcohol consumption were cost saving, and that the individually targeted interventions assessed were cost-effective approaches for preventing alcohol related diseases, injuries and mortality.

\section{Conclusion}

Our examples of the use of HIA in Copenhagen illustrate how different quantitative methodologies and models for HIA can be adapted to the relevant context and used to estimate the potential effects of interventions and policies affecting public health. 